\title{
Open Routed Energy Distribution Network Simulation for Maui Village Case Study
}

\author{
Alexander DUDKO ${ }^{\mathrm{a}}$, Tatiana ENDRJUKAITE ${ }^{\mathrm{b}}$, Leon ROOSE $^{\mathrm{c}}$ \\ ${ }^{a}$ KEIO University, Graduate School of Media and Governance, Kanagawa, Japan \\ ${ }^{\mathrm{b}}$ Transport and Telecommunication Institute, Research Department, Riga, Latvia \\ ${ }^{\mathrm{c}}$ Hawaiian Natural Energy Institute, University of Hawaii, Honolulu, US
}

\begin{abstract}
As the electricity generation is shifting to renewable energy sources (RES), the grid infrastructure faces multiple challenges, such as intermittency and volatility of a wide range RES. A high penetration of renewables requires profound changes to the current energy distribution system. The conventional grid is increasingly becoming a bottleneck for expanding the share of RES because of its rigid architecture, which is built around centralized energy source. We propose a new energy exchange model for a routed energy distribution system, which can perform electricity routing based on smart routing algorithms and presented protocols. We utilize a concept of an energy router device that controls energy flows and utilizes protocols stack to smartly route the energy between houses in the grid. This paper describes current results with experimental network of Maui village with multiple houses interconnected through energy routers.
\end{abstract}

Keywords: Energy distribution system, RES, Smart Grid, Energy Router.

\section{Introduction}

To support increasingly high penetration of intermittent renewables, such as solar and wind power generation, and distributed generation (DG), many regions are planning to add new high capacity transmission lines. These additional transmission lines strengthen grid synchronization but will also increase the grid's operation equipment requirements and protection complexity, and furthermore will be very costly.

The conventional approach of providing electricity from the point of generation, such as power plant, deals with a high power and therefore requires very powerful equipment. Every sub-station is responsible for providing power to designated region or area. If a sub-station goes down, the area is left without energy supply. Such case is highly undesirable, that is why to ensure energy supply there should be reserve substations or more complex fault-tolerant equipment. Modern energy grid has been developed with extensive interconnections with the purpose to improve reliability through redundancy. However, this interconnection increases the risk of wide area failures because any imbalance can be propagated quickly over an ever-widening area [1].

As the electricity generation is shifting to renewable sources, the grid infrastructure faces multiple challenges: intermittency and variability of a wide range of renewable 
sources, geographically distributed generation, bi-directional power flow and a need for energy storage systems (ESS) for meeting demand response requirements [2, 3, 4]. A high penetration of renewables requires profound changes to the current energy distribution system. The conventional grid is increasingly becoming a bottleneck for expanding the share of renewable energy sources (RES), because of its rigid architecture, which is built around centralized energy source.

Modern solutions such as renewables based distributed generation (DG) units, energy storage systems (ESSs), and advanced control strategies based on information and communication technologies have made possible for energy engineers and researchers to redesign the conventional power systems. However, not all these redesigning procedures are accomplished until now, as more researches are needed to make such advanced systems a reality $[5,6]$.

Modern world needs more than a set of new improvements. The need of a revolution of the energy generation and distribution is essentially important. The new system has to be redesigned from a scratch using all achievements in the field and to have a target to meet all the needs of today's and foreseeable future.

The direction of the future energy system needs a way to standardize the area to some extent and contribute to projects cooperation to move to the common goal. The new standard needs to be based on the common concepts so that different systems could be easily integrated together but be open for extensions, so that it does not limit the possibilities. And researches do not need to invent entire architecture complexity with all the details but focus only on specific aspects without the need to prove and explain all possible implementation variety.

This paper presents an alternative way of energy distribution. It is based on our recent research paper where we proposed a new energy exchange model for a routed energy distribution system, which can perform electricity routing based on smart routing algorithm and protocols [12]. We utilized a concept of an energy router device that uses energy as an input and protocols stack to smartly route an energy between houses in the grid.

It is a smart grid where electrical energy flows are controlled by routers. Such network is a combination of both power system and information communication system networks. Energy distribution and management system by means of the new concept of the energy routing aims to make a significant step forward into the next generation of electric grid and smart energy management.

This paper is organized as follows: Related work is presented in Section 2. Section 3 describes flows calculation approach. Experiments results are presented in Section 4. Section 5 presents conclusions of this work.

\section{Related Work}

To the best of our knowledge, there is not much work on the next generation energy smart grids in the world today that radically shifts the vision of electric grids and significantly changes the topology of the energy network.

However, during the last two decades researchers started to explore alternatives ways for energy systems. Several attempts have been done by researchers to propose distributed energy generation concepts for RES and new architectures for the energy distribution. Some researches try to reuse most of the existing infrastructure and simplify 
the transition into a new architecture [1,9], others require complete rebuild from the ground up but with integration option into existing electrical grid [10, 11].

Moreover, there are some ongoing research projects, like in Okinawa Island in Japan. There is a direct current (DC) based Open Energy System (DCOES) joint research project. This project is researching on a DC-based, bottom-up system that generates, stores, and shares electrical energy. Every house is equipped with PV panels, batteries, and an energy exchange system. These houses are interconnected via DC networks. It is designed to use the combination of various renewable energy sources and is expected to be deployed in various types of communities [7].

Annette Werth et al. analyzed a new type of DC-based, distributed interconnection of DC nanogrids. In her paper she proposed a new concept, both in terms of hardware and software architecture and showed the benefits on four-node simulations using physical model. Werth noted that the research is ongoing and some parts of the concepts still need to be studied [8].

DC power network with the concept of micro grid (MG) can provide power supply only to a restricted area, such as a small village, or building, for example a university campus. However, it is impossible to shift from AC to DC network globally and make DC power network a standard for commercial energy systems as it was done with the AC power network. Nowadays there are centralized power stations and electricity has to be transmitted over long distances.

Moreover, currently RES have installations limitations in the modern power grid due to two main reasons. First, backfeeding energy at peak times can break transformer and other equipment in the grid, when generated energy amounts are too high. Second, if baseline power plant generation goes too low when RES provide enough energy it becomes a risk, because power plant ramp rate might be not enough to quickly provide high power; for example when clouds covers all photovoltaic (PV) panels in the grid and RES generation is suddenly stopped.

\section{Approach}

This chapter describes the flows calculation approach and discusses the convergence of the entire system by continuously adapting the behavior of routers individually. A simple model simulation and results represent the important feature of the system to automatically reorganize the flows in case of broken transmission lines.

\subsection{Flows calculation}

The proposed energy exchange model is built on an idea of storage units and flows between them where energy routers represent a virtual storage unit and electrical lines in the network carry the flows. Every router $R_{i}$ has its level $L_{i}$, input and output flows $F\left(R_{i}, R_{j}\right)$ with connected router $R_{j}$. Directly connected routers have an energy flow to equalize their levels as shown in (1), where $\mathrm{k}$ is a flow sharing coefficient. Differential equation (2) shows how the router level depends on flows connected to router $R_{i}$.

$$
\begin{gathered}
F\left(R_{i}, R_{j}\right)=k \times\left(L_{i}-L_{j}\right) \\
d L_{i}=\sum_{j=1}^{N} F\left(R_{i}, R_{j}\right)
\end{gathered}
$$


Energy flow $F\left(R_{a}, R_{b}\right)$ is positive if energy is flowing from router $R_{a}$ to router $R_{b}$ and is negative if the direction is opposite, so the $F\left(R_{a}, R_{b}\right)=-F\left(R_{b}, R_{a}\right)$.

For a more realistic case the energy flow has to be limited according to physical constraints of the wire which links the two routers, so the flow equation becomes as shown in equations (3.1) and (3.2).

$$
\begin{gathered}
F\left(R_{i}, R_{j}\right)=g\left(k \times\left(L_{i}-L_{j}\right), F_{\text {max }}\left(R_{i}, R_{j}\right)\right) \\
g(x, m)=\left\{\begin{array}{cc}
-m, & x<-m \\
x & , \quad-m \leq x \leq m \\
m & , \quad x>m
\end{array}\right.
\end{gathered}
$$

\subsection{System Convergence}

The entire system is converging to satisfy all demands. The router levels $L$ do not become equal. Instead, the levels get values different to amount of flows between routers, and the system keep dynamically adjusting to the changing energy demands.

System convergence speed depends on the network topology, number of routers and links between them, as well as depends of the demand values from the customers. On the other side, the model has a flow sharing coefficient $k$ which can be chosen specifically for a given static configuration of the network. The lower is the value of $\mathrm{k}$, the slower the system gets to the convergence. If the coefficient is chosen too high, the model may not get to the convergence. Figure 1 shows how model reaches convergence given the different values of the $k$.

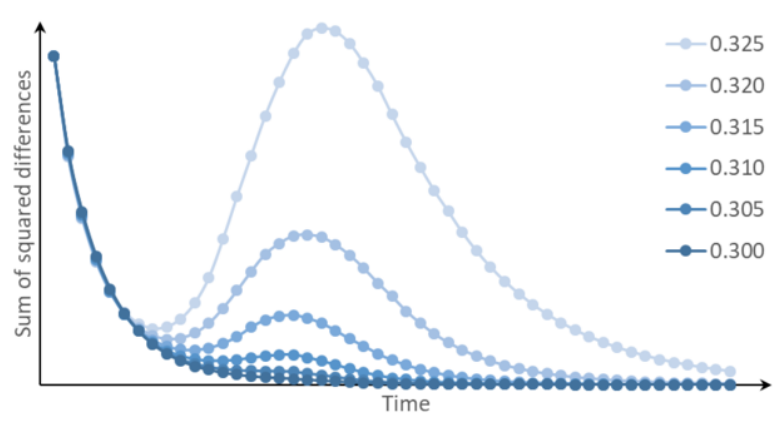

Figure 1. Convergence of the system depending on different flow sharing coefficient $\mathrm{k}$.

For a case of a relatively small network with multiple routers where consumers are located many hops away from energy generators a notable delay can appear between the moment of demand and the moment when energy is supplied. It happens because the demand gradually propagates through routers path to the generator and then gradually the energy supply makes its way back to the consumer.

In a more populated network the energy flow is constantly high, so individual consumers' power demand variations are small compared to overall network demand and they can be supplied immediately at the cost of little "power drop" for all neighbors for a short period until the system converges again. 
Convergence factor is a changeable parameter and it has to maintain the balance between the responsiveness and stability. On one side it specifies the convergence speed, on the other side it prevents energy to travel between routers in arbitrary way due to quick demand fluctuations.

\subsection{Grid Model and Simulation}

The considered case with 20 houses in a village and one power station connected to a routed smart grid was chosen as a model for simulation and experiments. In the basic simulation every house demands $5 \mathrm{~kW}$ of energy. The goal of the simulation was to determine the energy flows between routers (R1-R7), houses, and the power station S. Figure 2 shows the simulation result. Arrows in the figure show flows directions and amounts in $\mathrm{kW}$.

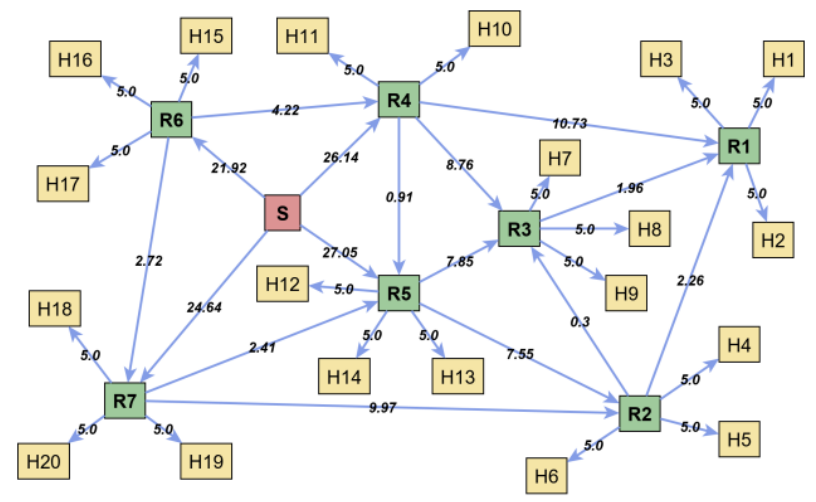

Figure 2. Model simulation result for experiment 1.

Table 1 shows a simulation result for the experimental model. All houses are receiving the demanded energy flows from connected routers. The second part of the experiment shows what happens when a single connection line breaks.

Table 1. Energy flows in experimental village model.

\begin{tabular}{|c|c|c|c|c|c|c|c|c|}
\hline $\mathrm{S}$ & R1 & R2 & $\mathrm{R} 3$ & R4 & R5 & R6 & R7 & $\mathrm{H}$ \\
\hline $\mathrm{S}$ & 0.00 & 0.00 & 0.00 & 26.14 & 27.05 & 21.92 & 24.64 & \\
\hline 0.00 & & -2.26 & -1.96 & -10.73 & 0.00 & 0.00 & 0.00 & 15.0 \\
\hline 0.00 & 2.26 & & 0.30 & 0.00 & -7.55 & 0.00 & -9.97 & 15.0 \\
\hline 0.00 & 1.96 & -0.30 & & -8.76 & -7.85 & 0.00 & 0.00 & 15.0 \\
\hline-26.14 & 10.73 & 0.00 & 8.76 & & 0.91 & -4.22 & 0.00 & 10.0 \\
\hline-27.05 & 0.00 & 7.55 & 7.85 & -0.91 & & 0.00 & -2.41 & 15.0 \\
\hline-21.92 & 0.00 & 0.00 & 0.00 & 4.22 & 0.00 & & 2.72 & 15.0 \\
\hline-24.64 & 0.00 & 9.97 & 0.00 & 0.00 & 2.41 & -2.72 & & 15.0 \\
\hline $\mathrm{H}$ & -15.0 & -15.0 & -15.0 & -10.0 & -15.0 & -15.0 & -15.0 & \\
\hline
\end{tabular}

The results after switching down line S-R5 is shown in Table 2. The system adapted to deliver energy to customers through new paths passing the broken line. 
Table 2. Village model with changes after disabling line between $\mathrm{S}$ and R5.

\begin{tabular}{|c|c|c|c|c|c|c|c|c|c|}
\hline & $\mathrm{S}$ & R1 & R2 & R3 & R4 & R5 & R6 & R7 & $\mathrm{H}$ \\
\hline S & & 0.00 & 0.00 & 0.00 & 36.09 & 0.00 & 28.33 & 34.03 & \\
\hline R1 & 0.00 & & -1.15 & -0.74 & -12.84 & 0.00 & 0.00 & 0.00 & 15.0 \\
\hline R2 & 0.00 & 1.15 & & 0.41 & 0.00 & -2.56 & 0.00 & -13.74 & 15.0 \\
\hline R3 & 0.00 & 0.74 & -0.41 & & -12.09 & -2.97 & 0.00 & 0.00 & 15.0 \\
\hline R4 & -36.09 & 12.84 & 0.00 & 12.09 & & 9.12 & -7.76 & 0.00 & 10.0 \\
\hline R5 & 0.00 & 0.00 & 2.56 & 2.97 & -9.12 & & 0.00 & -11.18 & 15.0 \\
\hline R6 & -28.33 & 0.00 & 0.00 & 0.00 & 7.76 & 0.00 & & 5.71 & 15.0 \\
\hline R7 & -34.03 & 0.00 & 13.74 & 0.00 & 0.00 & 11.18 & -5.71 & & 15.0 \\
\hline $\mathrm{H}$ & & -15.0 & -15.0 & -15.0 & -10.0 & -15.0 & -15.0 & -15.0 & \\
\hline
\end{tabular}

From the result we can see that all consumers receive demanded energy $5 \mathrm{~kW}$, total output flow from the power station $\mathrm{S}$ is equal to the total consumed power by all consumers and is $100 \mathrm{~kW}$. What is important to mention is that the energy distribution configuration was not calculated in a single place, but instead every router was performing local simple calculations and none of the routers has information about the network topology, size, or any other configuration. So, the solution for a complex system is getting found through a swarm intelligence (SI) where every individual performs simple operations and calculations.

\section{Experiments}

In this chapter we describe experiment of the proposed routed energy distribution network concept in a part of Maui village, located in Hawaii islands, USA. The village part of 37 houses is connected to utility power line station was chosen as a model for simulation and experiments. For the simulation every house demand was measured for 24 hours with discretization by 1 second.

\subsection{Existing Power Lines}

The considered case is based on a part of village in Maui, an isolated island in Hawaii, USA.

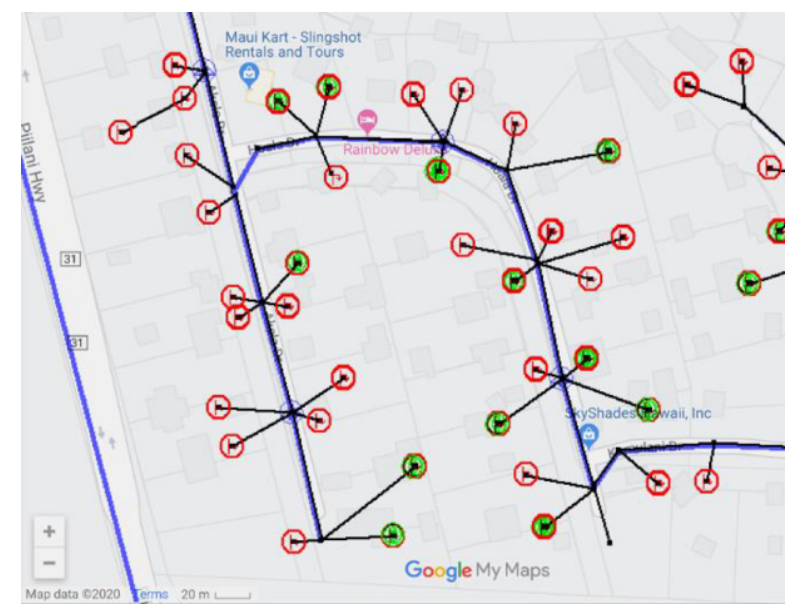

Figure 3. Existing power lines. 
The village part of 37 houses connected to utility power line station was chosen as a model for simulation and experiments. Every house demand was measured for 24 hours with discretization by 1 second. Figure 2 shows the existing power lines provided by the electrical utility company and connections to houses.

\subsection{Maui Village Model}

In this experiment a village model was created based on the existing power lines and houses connection to closely simulate a real usage scenario. The goal of the simulation was to show the operation of the system throughout the 24 hours and to determine the energy flows between routers R1-R10, houses H1-H37, and the power station presented as a generator G1. The model is shown in Figure 4.

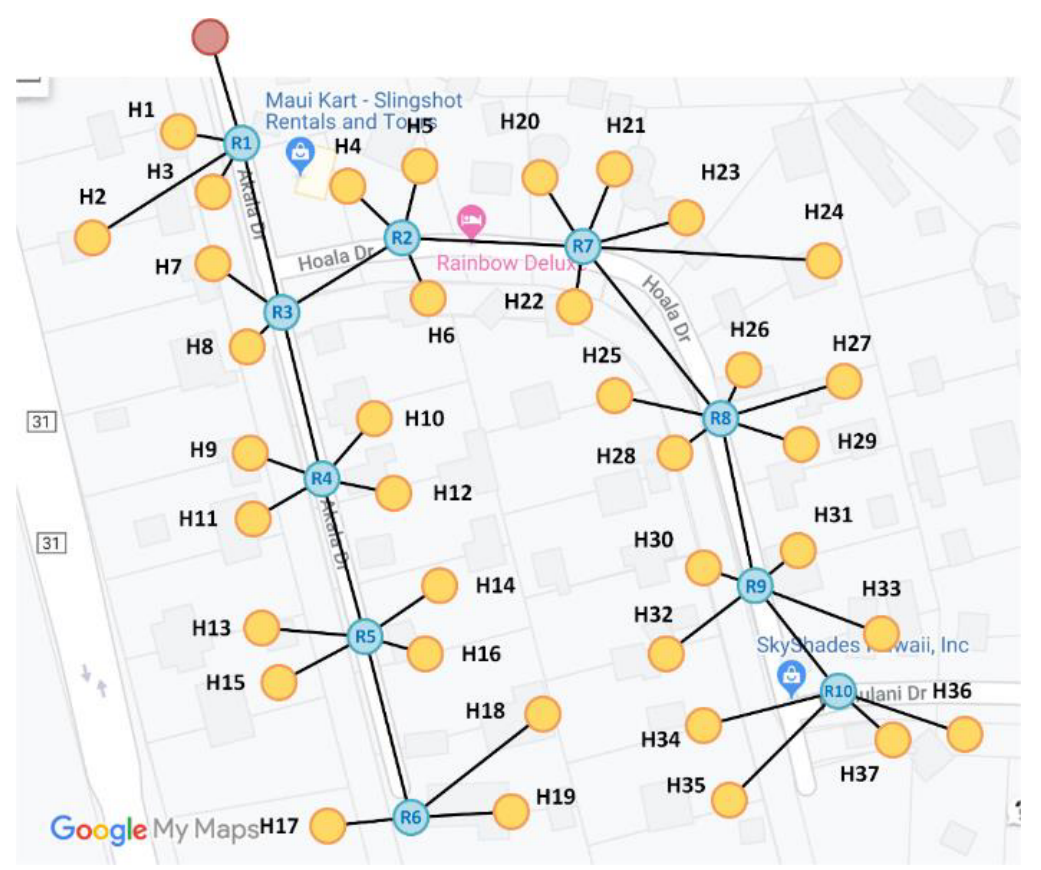

Figure 4. Maui village model.

Figure 5 shows a typical house load data (a) and its breakdown into components which shows own consumer load (b) with a periodic component (c), and high frequency fluctuations (d) which are observable equal for all houses within a network.

Figure 6 shows 5-minute fragment of the simulation result which shows how the network reacts to high-frequency fluctuations in the houses loads. The model shows that fast fluctuations and jitter are not propagating much with a network affecting only local segments. Network smooth out any quick fluctuations which makes the system stable and resistant to noise. 
(a)

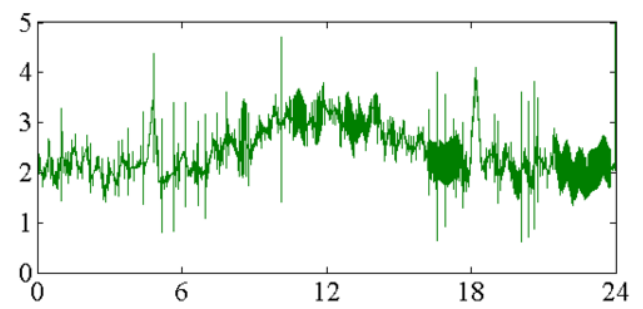

(b)

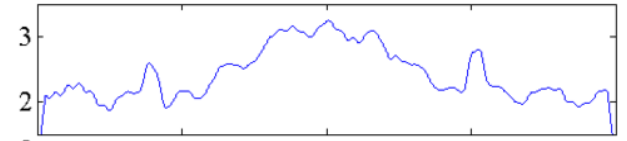

(c)

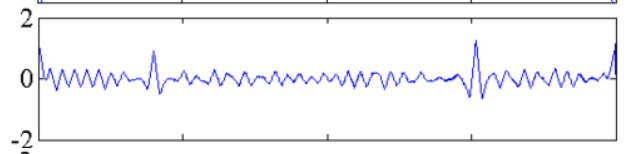

(d)

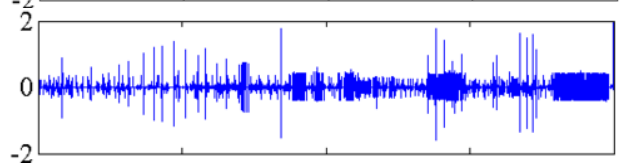

Figure 5. Breakdown of a load signal from a real world consumer.

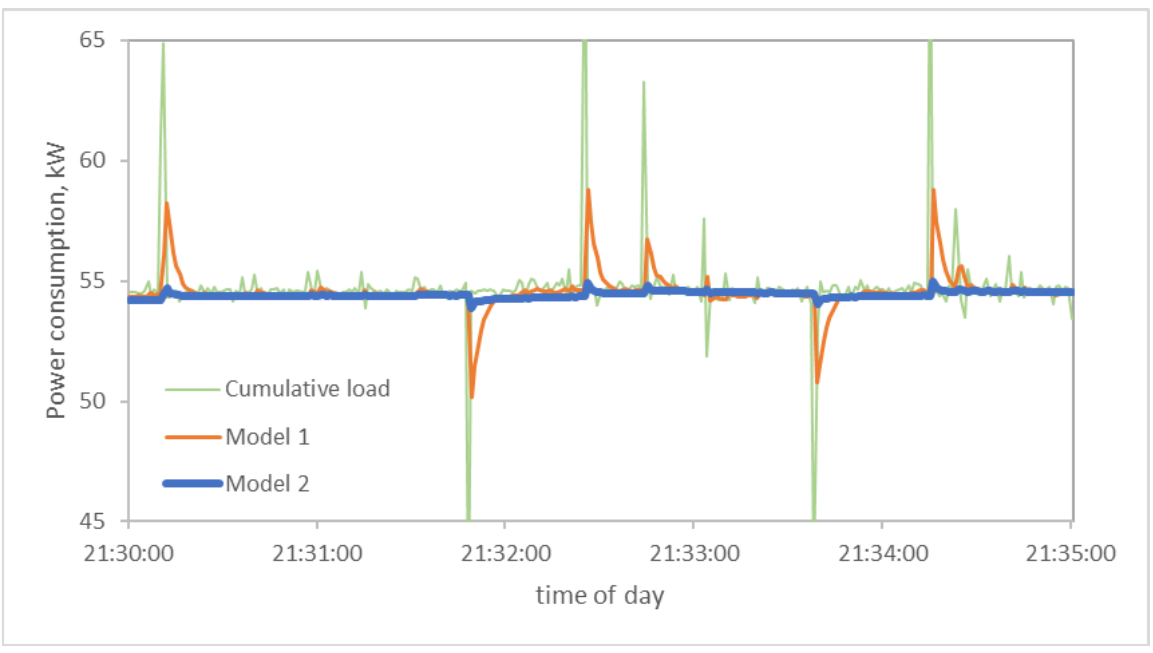

Figure 6. 5-minute fragment of the simulation with high-frequency fluctuations.

Table 3 shows total amount of energy passed by connection lines in kWh for simulation period of 24 hours. We can clearly see that full amount of energy is passing through the router R1 which is the only path between the village and the power plant substation. Every further line down passes less and less energy delivering it houses down the line. 
Table 3. Total amount of energy flows between routers and houses of the network in $\mathrm{kWh}$.

\begin{tabular}{|c|c|c|c|c|c|c|c|c|c|c|c|c|}
\hline & G1 & R1 & R2 & R3 & R4 & R5 & R6 & R7 & R8 & R9 & R10 & $\mathrm{H}$ \\
\hline G1 & & 965 & 0 & 0 & 0 & 0 & 0 & 0 & 0 & 0 & 0 & \\
\hline R1 & -965 & & 0 & 832 & 0 & 0 & 0 & 0 & 0 & 0 & 0 & 133 \\
\hline $\mathrm{R} 2$ & 0 & 0 & & -517 & 0 & 0 & 0 & 444 & 0 & 0 & 0 & 73 \\
\hline R3 & 0 & -832 & 517 & & 274 & 0 & 0 & 0 & 0 & 0 & 0 & 41 \\
\hline $\mathrm{R} 4$ & 0 & 0 & 0 & -274 & & 109 & 0 & 0 & 0 & 0 & 0 & 165 \\
\hline R5 & 0 & 0 & 0 & 0 & -109 & & 48 & 0 & 0 & 0 & 0 & 62 \\
\hline R6 & 0 & 0 & 0 & 0 & 0 & -48 & & 0 & 0 & 0 & 0 & 48 \\
\hline R7 & 0 & 0 & -444 & 0 & 0 & 0 & 0 & & 381 & 0 & 0 & 63 \\
\hline $\mathrm{R} 8$ & 0 & 0 & 0 & 0 & 0 & 0 & 0 & -381 & & 242 & 0 & 139 \\
\hline R9 & 0 & 0 & 0 & 0 & 0 & 0 & 0 & 0 & -242 & & 147 & 96 \\
\hline R10 & 0 & 0 & 0 & 0 & 0 & 0 & 0 & 0 & 0 & -147 & & 147 \\
\hline $\mathrm{H}$ & & -133 & -73 & -41 & -165 & -62 & -48 & -63 & -139 & -96 & -147 & \\
\hline
\end{tabular}

\subsection{Distributed Generation}

Since the penetration of renewable energy resources increases, the need to effectively manage these resources in distribution networks also increases. By means of Energy Routers more renewables can be installed compared to the conventional networks. Customers are not limited in the power of renewable energy generation units and their amount in the network. The house owners are able to use own generated energy, and the energy router is spreading the remaining energy between other routers as much as they can accept.

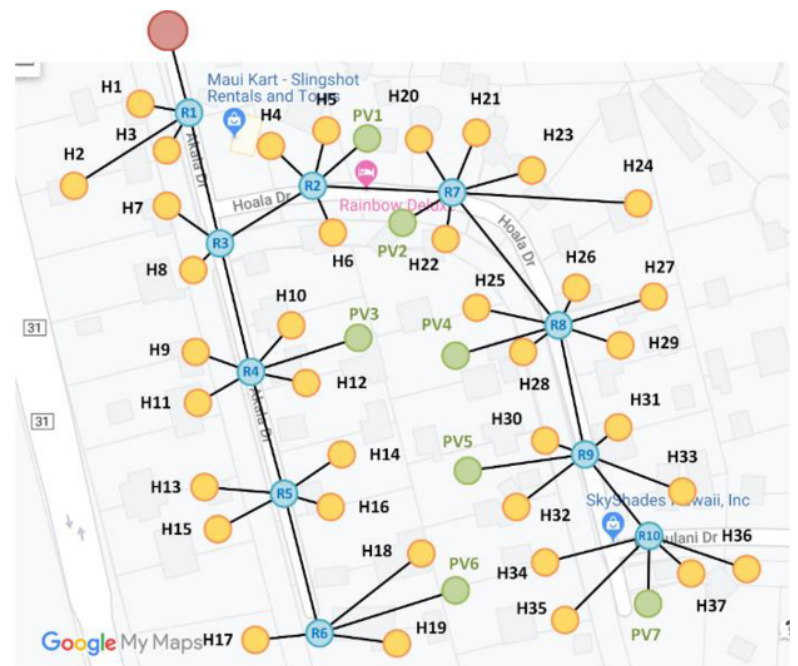

Figure 7. New network configuration with PV.

This experiment includes 7 photovoltaic (PV) generators in the model very close to how these are installed in the existing houses. PV panels generation was taken based on measured values for 24 hours data with discretization of 1 value per second. Figure 7 shows locations of PV panels and new network configuration for total of 55 nodes. 
The results of simulation are shown on Table 4. The values are much lower compared to previous experiment shown in Table 3, because the energy was mostly consumed locally, therefore reducing the high energy flows throughout the entire network.

Table 4. Total amount of energy flows between routers and houses of the network in $\mathrm{kWh}$ with PV.

\begin{tabular}{c|ccccccccccccc|}
\multicolumn{1}{c}{} & $\mathrm{G} 1$ & $\mathrm{R} 1$ & $\mathrm{R} 2$ & $\mathrm{R} 3$ & $\mathrm{R} 4$ & $\mathrm{R} 5$ & $\mathrm{R} 6$ & $\mathrm{R} 7$ & $\mathrm{R} 8$ & $\mathrm{R} 9$ & $\mathrm{R} 10$ & $\mathrm{H}$ & PV \\
\cline { 2 - 14 } G1 & & 141 & 0 & 0 & 0 & 0 & 0 & 0 & 0 & 0 & 0 & & \\
\cline { 2 - 15 } R1 & -141 & 0 & 0 & 8.1 & 0 & 0 & 0 & 0 & 0 & 0 & 0 & 133 & 0 \\
R2 & 0 & 0 & 0 & 43 & 0 & 0 & 0 & -11 & 0 & 0 & 0 & 73 & -105 \\
R3 & 0 & -8.1 & -43 & 0 & 10 & 0 & 0 & 0 & 0 & 0 & 0 & 41 & 0 \\
R4 & 0 & 0 & 0 & -10 & 0 & 4.2 & 0 & 0 & 0 & 0 & 0 & 165 & -159 \\
R5 & 0 & 0 & 0 & 0 & -4.2 & 0 & -58 & 0 & 0 & 0 & 0 & 62 & 0 \\
R6 & 0 & 0 & 0 & 0 & 0 & 58 & 0 & 0 & 0 & 0 & 0 & 48 & -105 \\
R7 & 0 & 0 & 11 & 0 & 0 & 0 & 0 & 0 & 21 & 0 & 0 & 63 & -95 \\
R8 & 0 & 0 & 0 & 0 & 0 & 0 & 0 & -21 & 0 & -2 & 0 & 139 & -116 \\
R9 & 0 & 0 & 0 & 0 & 0 & 0 & 0 & 0 & 2 & 0 & 16 & 96 & -114 \\
R10 & 0 & 0 & 0 & 0 & 0 & 0 & 0 & 0 & 0 & -16 & 0 & 147 & -130 \\
H & & -133 & -73 & -41 & -165 & -62 & -48 & -63 & -139 & -96 & -147 & & \\
PV & & 0 & 105 & 0 & 159 & 0 & 105 & 95 & 116 & 114 & 130 & & \\
\hline
\end{tabular}

Ultimately, the more people install own energy sources such as PV panels the less network depends on the power station energy. The area or a village with many RES generators and ESSs becomes more independent in this case. Central power station becomes less important and less used. Instead the whole network becomes a decentralized virtual power plant (VPP) with multiple small power sources which together can cover needs of the whole network.

\section{Conclusions}

In this paper we introduced a novel approach of energy distribution based on routing. The proposed Routed Smart Grid system has a lot of advantages compared to conventional energy systems widely used nowadays: better scalability, because conventional energy distribution networks are not flexible in terms of scaling. Equipment need to be changed when the network grows bigger and bigger. Proposed Routed Smart Grid system easily scales by simply connecting and disconnecting nodes anywhere in the network; More stable source load, when we are using energy routers the network has a potential to have a more stable network load; Higher reliability of the system and better fault tolerance, because there are many ways how energy can be provided to the every consumer, the damage of routers or wires will not cause the network to stop operate; Greater usage of renewable energy sources, by means of energy routers, we can install more renewables compared to the system without routers. Routers give smart control over energy flows. We can easily measure, route, and limit the amounts of energy for any node in the system. 


\section{Acknowledgement}

This work was financially supported by the specific support objective activity 1.1.1.2. "Post-doctoral Research Aid" (Project id. N. 1.1.1.2/16/I/001) of the Republic of Latvia, funded by the European Regional Development Fund. Tatiana Endrjukaite research project No. 1.1.1.2/VIAA/1/16/095 "Integrated Model for Energy Generation, Distribution and Management".

\section{References}

[1] R. Abe, H. Taoka, D. McQuilkin. Digital grid: communicative electrical grids of the future. IEEE Transactions on Smart Grid 2011; 2(2): 399-410.

[2] A. Werth, N. Kitamura, M. Tokoro, K. Tanaka. Evaluation model for multi-microgrid with autonomous DC energy exchange. IEEJ Transactions on Electrical and Electronic Engineering 2017 12(5): 676-682.

[3] H. Farhangi, The path of the smart grid. IEEE Power and Energy Magazine 2010; 8: 18-28.

[4] P. Khayyer and U. Ozguner. Decentralized Control of Large-Scale Storage-Based Renewable Energy Systems. IEEE Transactions on Smart Grid 2014; 5: 1300-1307.

[5] J. Justo, F. Mwasilu, J. Lee, J. Jung. AC-microgrids versus DC-microgrids with distributed energy resources: A review. 2013. Renewable and Sustainable Energy Reviews, vol. 24, (2013), 387-405.

[6] P. Asmus. 2010. Microgrid, virtual power plants and our distributed energy future. The Electricity Journal, vol. 23, no. 10 (2010), 72-82.

[7] M. Tokoro. 2014. Sony CSL-OIST DC-based open energy system (DCOES). In Proc. of the 1st International Symposium on Open Energy Systems (Okinawa, Japan, 2014), 64-67.

[8] A. Werth, N. Kitamura, K. Tanaka. 2015. Conceptual Study for Open Energy Systems: Distributed Energy Network Using Interconnected DC Nanogrids. IEEE Transactions on Smart Grid, vol. 6, no. 4, (Jul. 2015), $1621-1630$.

[9] T. Nguyen-Van, R. Abe. K. Tanaka. MPPT and SPPT Control for PV-Connected Inverters Using Digital Adaptive Hysteresis Current Control. Energies 2018, 11, 2075.

[10] T. Kaipia, P. Salonen, J. Lassila, J. Partanen. Application of low voltage DC-distribution system: a techno-economical study. In Proc. of 19th Int. Conf. on Electricity Distribution, Vienna, Austria, 2007, $1-4$.

[11] T. Sakagami et al. Performance of a DC-based microgrid system in Okinawa. In Proc. of International Conference on Renewable Energy Research and Applications 2015.

[12] T. Endrjukaite, A. Dudko, L.Roose. Energy Exchange Model in Routed Energy Distribution Network. In Proc. of the 6th ACM International Conference on Systems for Energy-Efficient Buildings, Cities, and Transportation (ACM BuildSys 2019), New York, US, November 2019, 393-394. 